\section{A sensibilidade antibiótica do pneumococo $e$ o tratamento da pneumonia na criança}

\section{Prezado Editor,}

Com a atual epidemia de infecções por cepas de Streptococcus pneumoniae parcialmente resistentes à penicilina (PRSP), tem surgido algumas recomendações de se elevar a dose de ampicilina de $100 \mathrm{mg} / \mathrm{kg}$ para $150 \mathrm{mg} / \mathrm{kg}$ no tratamento das pneumonias de aquisição comunitária na infância ${ }^{1}$. Essa conduta, além de onerar o custo terapêutico, teoricamente poderia concorrer para o agravamento da questão da resistência antimicrobiana, especificamente aqui, o S. pneumoniae versus a penicilina. Mas haveria evidências científicas suficientes para embasar tal procedimento? Parece que não, como veremos a seguir.

Apesar do registro crescente em várias partes do mundo do PRSP, não se tem observado aumento nos índices de insucesso no tratamento das pneumonias de aquisição comunitária pelo $S$. pneumoniae. Essas evidências têm sido apontadas em diversas publicações, em diferentes regiões do mundo. Na Espanha, por exemplo, Pallares e colaboradores ${ }^{2}$, ao longo de 10 anos de observação em Barcelona, não detectaram alterações das taxas de mortalidade por pneumonia grave entre os pacientes infectados por cepas sensíveis ou resistentes à penicilina, tratados com ampicilina ou penicilina. Estudo multicêntrico nos Estados Unidos ${ }^{3}$, envolvendo 8 hospitais durante 3 anos, acompanhou 257 episódios de pneumonia, com 14\% de PRSP ou PPRSP. Não foram detectadas diferenças em relação à apresentação clínica ou ao desfecho, em relação às crianças com infecções com $S$. pneumoniae, sensíveis ou resistentes à penicilina. Kaplan e colaboradores ${ }^{4}$, em estudo prospectivo, com 1.255 crianças portadoras de infecção pneumocócica invasiva, observaram que dos 19 óbitos registrados, não havia diferenças entre os pacientes infectados por cepas sensíveis ou resistentes à penicilina. Nos 3 anos de duração de estudo, o número de cepas resistentes chegou a dobrar. Friendland e colaborado$\mathrm{res}^{5}$, em estudo na África do Sul, envolvendo 78 crianças com pneumonia pneumocócica ( 53 cepas sensíveis e 25 resistentes à penicilina), também não observaram diferenças em relação ao desfecho.

Nos Estados Unidos, apesar do S. pneumoniae persistir como a principal causa de pneumonia e com índices crescentes de resistência à penicilina (incidência de 27,8\% de PRSP e 16,0\% de resistência elevada - concentração inibitória mínima de MIC > $2 \mu \mathrm{g} / \mathrm{ml}$ ), a Sociedade Americana de Doenças Infecciosas afirma que o não conhecimento de falhas terapêuticas no tratamento dessas pneumonias, até a presente data, não os autoriza à realização de mudanças dos esquemas antibióticos empregados no tratamento das pneumonias adquiridas na comunidade ${ }^{6}$.

Para Harwell \& Brown ${ }^{7}$, em atualização recente, as doses habituais da penicilina e seus derivados ainda são eficazes para as infecções por RPSP. Uma das possíveis explicações para justificar essas diferenças de respostas in vivo das in vitro parece se encontrar na farmacodinâmica da penicilina. Nos pulmões, os alvéolos e a grande massa capilar são separados apenas pela espessura de duas células que chegam a dividir uma mesma membrana basal. Isso permite que as concentrações do antibiótico no alvéolo sejam bastante aproximadas das do sangue, especialmente nas situações em que a presença do processo inflamatório acarrete alterações fisiológicas e anatômicas ${ }^{8}$. Já nas infecções intravasculares, como as endocardites, ou naquelas em que a penetração do antibiótico é restrita, como nas meningites, a concentração plasmática do antibiótico parece essencial.

Um outro ponto a ser considerado no tratamento das pneumonias é o de que os estudos são baseados em hemoculturas, o que provavelmente envolve cepas invasivas. Em mais da metade dos casos de pneumonia de aquisição comunitária, as hemoculturas são negativas.

Dessa forma, acreditamos que a mudança de conduta no tratamento das pneumonias pneumocócicas em crianças, de aquisição comunitária, deva ser baseada em ensaios clínicos e não em resultados laboratoriais in vitro. Como nas bases de dados pesquisadas (Medline, Lilacs) ainda não estão registrados esses ensaios que poderiam dar suporte ao aumento das doses da ampicilina no tratamento das pneumonias de aquisição comunitária, a melhor evidência científica é manter as doses empregadas atualmente.

\section{Referências bibliográficas}

1. Ruvinski R, Balanzal MC. In: Benguigui Y, Antuñano FJ, Schmunis G, Yunes J. Infecções respiratórias em crianças. Washington, D.C.: OPAS; 1998.

2. Pallares R, Liñares J, Vadillo M, Labelos C, Manresa F, Viladrich $\mathrm{PF}$, et al. Resistance to penicillin and cephalosporin and mortality from severe pneumococcal pneumonia in Barcelona, Spain. $\mathrm{N}$ Engl J Med 1995;333:474-80.

3. Tan TQ, Mason EO Jr, Barson WJ, Wald ER, Schutze GE, Bradley JS, et al. Clinical characteristics and outcome of children with pneumonia atrributable to penicillin-susceptible and penicillin-nonsusceptible Streptococcus pneumoniae. Pediatrics 1998;102:1369-75. 
4. Kaplan SL, Mason EO Jr, Barson WJ, Wald ER, Arditi M, Tan $\mathrm{TQ}$, et al. Three-year multicenter surveillance of asystemic pneumococcal infections in children. Pediatrics 1998;102:538-45.

5. Friedland IR. Comparison of the response to antimicrobial therapy of penicillin-resistant and penicillin-susceptible pneumococcal disease. Pediatr Infect Dis J 1995 ;14(10):885-90.

6. Bartlett JG, Breiman RF, Mandell LA, File TM Jr. Communityacquired pneumonia in adults: guidelines for management; The Infectious Diseases Society of America. Clin Infect Dis 1998;28:811-38.

7. Harwell JI, Brown RB. The drug-resistant Pneumococcus. Clinical relevance, therapy, and prevention. Chest 2000;117:530-41.

8. Siegel PE. The significance of serum vs tissue levels of antibiotics in the treatment of penicillin-resistant Streptococcus pneumoniae and community-acquired pneumonia. Chest 1999;116:535-8.

\section{Dr. João Guilherme Bezerra Alves}

Prof. Adjunto da Faculdade de Ciências Médicas da UPE e do IMIP

\section{Prezado Editor,}

Tivemos a satisfação de ler no Jornal de Pediatria o artigo de Rey L.C. e cols. ${ }^{1}$, que avaliou o perfil de sensibilidade antibiótica de $S$. pneumoniae isolado em nasofaringe, comentado em editorial por Camargos P.A.M ${ }^{2}$. Os resultados apresentados indicam que a resistência do $S$. pneumoniae à penicicilina/amoxicilina é crescente no Brasil, como ocorre em todo o mundo ${ }^{3}$. Porém, a resistência plena a estas drogas situase em patamar limitado ${ }^{1}$. A ampliação da taxa de cepas resistentes decorreu, em nosso meio, em grande parte pelo incremento de bactérias parcialmente resistentes. Em estudo anterior, também observamos resultados assemelhados aos agora publicados, em avaliação de cepas de $S$. pneumoniae causadoras de pneumonia aguda ${ }^{4}$.

A implicação terapêutica destes resultados para as pneumonias da comunidade é o de manter a recomendação do uso da penicilina/amoxicilina no tratamento da pneumonia pneumocócica; em virtude das cepas parcialmente resistentes serem clinicamente responsivas a estas drogas ${ }^{5,6}$. A dosagem de penicilina/amoxicilina não está claramente estabelecida para o tratamento destas pneumonias. Alguns autores têm sugerido uma elevação da dosagem diária para 100.000 a 200.000 unidades $/ \mathrm{kg}$ de penicilina ou 70 a $90 \mathrm{mg} / \mathrm{kg} / \mathrm{dia}$ de amoxicilina $^{5,6}$. O incremento na dosagem da amoxicilina toma por base a recomendação para o tratamento da otite pneumocócica.

Outro aspecto que nos parece relevante no artigo apresentado foi o da resistência do $S$. pneumoniae a outros antimicrobianos, especialmente à eritromicina (23\%), como também se verifica em outros países ${ }^{3}$. Este resultado é preocupante, pois os macrolídeos têm sido recomendados para o tratamento de pneumonias comunitárias em duas faixas etárias, nos primeiros meses de vida (objetivando tratar infecção por Chlamydia trachomatis) e, principalmente, após os cinco anos de idade (visando às infecções por Mycoplasma pneumoniae e Chlamydia pneumoniae $)^{6}$. Considerando o importante papel etio- lógico do S. pneumoniae na pneumonia comunitária em todas as faixas etárias, exceto no período neonatal, parece-nos pouco adequado iniciar o tratamento desta infecção com macrolídeo, exceto quando as evidências clínico-epidemiológicas forem muito sugestivas de outros agentes infeccio$\operatorname{sos}^{3-6}$. Adicionalmente, falta comprovar em nosso meio uma incidência significativa de pneumonias comunitárias causadas por Mycoplasma pneumoniae e Chlamydia pneumoniae.

A partir destas observações, temos mantido no Instituto da Criança e na Divisão de Pediatria do HU-USP, a opção preferencial pela penicilina/amoxicilina no início de tratamento da pneumonia comunitária das crianças com idade superior a 30 dias; exceto quando houverem indicações clínicas e/ou radiológicas de que o agente causal possa ser insensível a estas drogas. Em particular, nas crianças com idade inferior a 3 anos iniciamos o tratamento da pneumonia comunitária com a amoxicilina; temos por objetivo oferecer cobertura, também, à maior parte dos Hemophilus sp, agente bacteriano ainda incidente (não capsulados), apesar dos progressos na vacinação específica 5 .

Certamente, as mudanças que têm ocorrido no perfil de sensibilidade do $S$. pneumoniae exigem vigilância, que tem sido feita através do SIREVA ${ }^{1}$. No momento, as recomendações terapêuticas para a pneumonia comunitária, podem, ainda, ser conservadoras, como se depreende do editorial de Camargos P.A.M. ${ }^{2}$ Com a majoritária utilização de penicilina/amoxicilina, teremos, de modo geral, um tratamento eficaz e pouco oneroso, além de retardar o desenvolvimento de resistência bacteriana aos antibióticos de aquisição mais recente.

\section{Referências bibliográficas}

1. Rey LC, Wolf B, Moreira JLB, Verhoef J, Farhat CK. S. pneumoniae isolados da nasofaringe de crianças sadias e com pneumonia: taxa de colonização e sensibilidade aos antimicrobianos. J Pediatr (Rio J) 2002:78:105-12.

2. Camargos PAM. Resistência do $S$. pneumoniae à penicilina $\mathrm{G}$ no Brasil: a ponta do iceberg. J Pediatr (Rio J) 2002;78:87-8.

3. McCracken GH Jr. Etiology and treatment of pneumonia in children in the United States. Pediatr Infect Dis J 2000;19:373-7.

4. Lotufo JPB, Ferreira LLM, Hein N, Gilio AE, Brandileone MCC, Ejzenberg B, et al. Tratamento das infecções pneumocócicas [carta]. J Pediatr (Rio J) 2000;76:251-2.

5. Nelson JD. Community-acquired pneumonia in children: guidelines for treatment. Pediatr Infect Dis J 2000;19:251-3.

6. Lerou PH. Lower respiratory tract infections in children. Curr Opin Pediatr 2001;13:200-6.

\section{Dr. João Paulo Lotufo}

Mestre em Pediatria. Divisão de Pediatria do HU-USP

\section{Dr. Bernardo Ejzenberg}

Livre Docente em Pediatria. Coordenador de Pesquisas e Publicações do HU-USP

\section{Dr. Joaquim Carlos Rodrigues}

Doutor em Pediatria. Médico Chefe do Grupo de Pneumologia Instituto da Criança 


\section{Resposta do autor}

\section{Prezado Editor,}

O estudo traz à discussão a eficácia da vigilância do padrão de resistência aos antimicrobianos de uma bactéria comunitária como o pneumococo, utilizando a estratégia de analisar as amostras colonizando a nasofaringe. Revelou entre outros que nossas creches são um importante ambiente para desenvolvimento de penicilino- e mesmo multi-resistência dos germes colonizantes ${ }^{1}$. Os pneumococos colonizantes não devem ser considerados como agentes etiológicos: o estudo da colonização é valido no aspecto epidemiológico da resistência bacteriana. Para estudar a resistência específica do pneumococo no processo infeccioso, é necessário isolá-lo a partir de materiais clínicos.

Com relação ao uso de amoxicilina oral no tratamento ambulatorial das pneumonias comunitárias, existe consenso de que as doses habituais de $50 \mathrm{mg} / \mathrm{kg} /$ dia são suficientes para tratar pneumococos cujas concentrações inibitórias mínimas (CIM) de penicilina são de $1-2 \mu \mathrm{g} / \mathrm{ml}^{2,3}$. Os critérios de farmacodinâmica/farmacocinética (tempo em que a concentração do antibiótico se mantém acima da CIM) de 40-50\% do intervalo entre as doses têm se revelado eficazes para avaliar o potencial bactericida da droga, e confirmam os achados clínicos $^{3,4}$. Pneumococos causadores de pneumonia com CIM de penicilina $\geq 4 \mu \mathrm{g} / \mathrm{ml}$ (resistência plena) são raros e, nesses casos, doses mais elevadas de penicilina/amoxicilina costumam ser eficazes ${ }^{5}$.

Outra questão importante é a elevada resistência dos pneumococos ao sulfametoxazol/ trimetoprim (SMX/TMP), em nosso estudo. A associação SMX/TMP é amplamente utilizada no tratamento ambulatorial das pneumonias. No Paquistão, a alta resistência ao SMX/TMP in vitro não influenciou a boa eficácia clínica da droga na pneumonia comunitária não complicada em comparação com a amoxicilina (13\% e $12 \%$ de fracasso terapêutico respectivamente) ${ }^{6}$.

A Sociedade Brasileira de Pediatria, em revisão consensual dos Departamentos de Infectologia e Pneumologia sobre tratamento das pneumonias na infância e adolescência ${ }^{3}$, contrariando orientação do Ministério da Saúde, não recomenda o uso de SMX/TMP no tratamento ambulatorial das pneumonias comunitárias não complicadas; no entanto, carecemos de estudos clínicos relevantes que demonstrem a ineficácia do SMX/TMP em relação à amoxicilina nessas condições.

A resistência plena do pneumococo à eritromicina de nosso estudo foi elevada (28\%), apesar de menos utilizada que o SMX/TMP em nosso meio. Diversos relatos reforçam o fato de que o tratamento empírico de síndromes febris ou infecções de vias aéreas superiores com SMX/TMP e macrolídeos acarretam o aumento da resistência do pneumococo a estas drogas e também à penicilina ${ }^{5}$.

\section{Referências bibliográficas}

1. Giebink GS. The prevention of pneumococcal disease in children. N Engl J Med 2001;345:1177-83.
2. Tan TQ, Mason Jr EO, Barson WJ, Wald ER, Schutze GE, Bradley JS, et al. Clinical characteristics and outcome of children with pneumonia attributable to penicillin-susceptible and penicillin-nonsusceptible Streptococcus pneumoniae. Pediatrics 1998;102:1369-75.

3. Nascimento-Carvalho CM, Souza-Marques HH. Recomendação da Sociedade Brasileira de Pediatria para antibioticoterapia de crianças e adolescentes com pneumonia comunitária. Disponível em: http//:www.sbp.com.br/img/Pediat_doc.PDF, 2002.

4. Dagan R, Klugman KP, Craig WA, Baquero F. Evidence to support the rationale that bacterial erradication in respiratory tract infections is an important aim of antimicrobial therapy. $\mathbf{J}$ Antimicr Chemother 2001;47:129-40.

5. Klugman KP. Bacteriological evidence of antibiotic failure in pneumococcal lower respiratory tract infections. Eur Respir J Suppl 2002;36:3s-8s.

6. Strauss WL, Qazi SA, Kundi Z, Nomani NK, Schwartz B, and The Pakistan Co-trimoxazole Study Group. Antimicrobial resistance and clinical effectiveness of co-trimoxazole versus amoxycillin for pneumonia among children in Pakistan: randomised controlled trial. Lancet 1998;352:270-4.

\section{Dr. Luís C. Rey}

Pediatra, Doutor. Unidade de Pesquisa Clínica, Hospital Universitário, Universidade Federal do Ceará, Fortaleza, CE.

E-mail: reyfonte@secrel.com.br 